\title{
Attacks on Hash Functions Based on Generalized Feistel: Application to Reduced-Round Lesamnta and SHAvite- $3_{512}{ }^{\star}$
}

\author{
Charles Bouillaguet $^{1}$, Orr Dunkelman ${ }^{2}$, \\ Gaëan Leurent ${ }^{1}$, and Pierre-Alain Fouque ${ }^{1}$ \\ ${ }^{1}$ École normale supérieure \\ \{charles.bouillaguet, gaetan.leurent,pierre-alain.fouque\}@ens.fr \\ ${ }^{2}$ Weizmann Institute of Science \\ orr.dunkelman@weizmann.ac.il
}

\begin{abstract}
In this paper we study the strength of two hash functions which are based on Generalized Feistels. We describe a new kind of attack based on a cancellation property in the round function. This new technique allows to efficiently use the degrees of freedom available to attack a hash function. Using the cancellation property, we can avoid the non-linear parts of the round function, at the expense of some freedom degrees.

Our attacks are mostly independent of the round function in use, and can be applied to similar hash functions which share the same structure but have different round functions. We start with a 22-round generic attack on the structure of Lesamnta, and adapt it to the actual round function to attack 24-round Lesamnta (the full function has 32 rounds). We follow with an attack on 9-round SHAvite-3512 which also works for the tweaked version of SHAvite-3512.
\end{abstract}

\section{Introduction}

Many block ciphers and hash functions are based on generalized Feistel constructions. In this paper we treat such generalized Feistel constructions and especially concentrate on the case where an $n$-bit round function is used in a $4 n$-bit structure. Two of these constructions, shown at Figure 11 used in the Lesamnta and the SHAvite- $3_{512}$ hash functions, are the main focus of this paper.

While in the ideal Luby-Rackoff case, the round functions are independent random functions, in practice, most round functions $F(k, x)$ are usually defined as $P(k \oplus x)$, where $P$ is a fixed permutation (or function). Hence, we introduce several attacks which are based on cancellation property: if the fixed function $P$ accepts twice the same input, it produces twice the same output. In a hash

\footnotetext{
* The full version of this paper appears as IACR ePrint report 2009/634 3.

${ }^{1}$ Note that the direction of the rotation in the Feistel structure is not really important: changing the rotation is equivalent to considering decryption instead of encryption.
}

A. Biryukov, G. Gong, and D.R. Stinson (Eds.): SAC 2010, LNCS 6544, pp. 18 35, 2011.

(C) Springer-Verlag Berlin Heidelberg 2011 


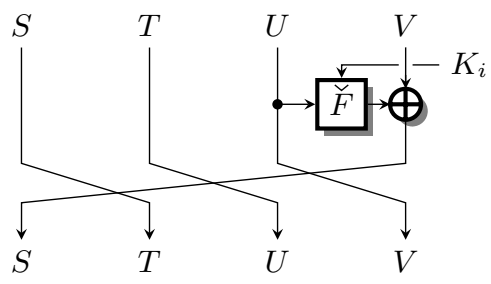

Lesamnta structure

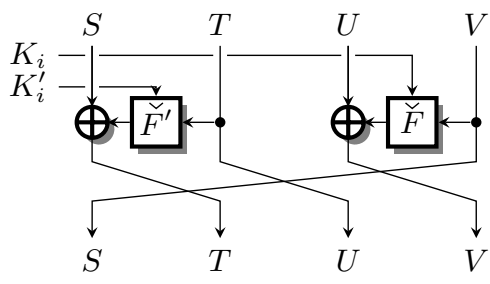

SHAvite-3512 structure

Fig. 1. The Generalized Feistel Constructions Studied in this paper

function setting, as there is no secret key, the adversary may actually make sure that the inputs are the same.

For Lesamnta we start with generic attacks that work independent of the actual $P$ in use, but then use the specific properties of Lesamnta's round functions to offer better attacks. The attack on SHAvite- $_{512}$ is a more complicated one, following the more complex round functions (and the structure which uses two functions in each round), but at the same time, is of more interest as SHAvite-3 ${ }_{512}$ is still a SHA3 candidate.

\subsection{Overview of the Attacks}

Our attacks are based on a partial preimage attack, i.e. we can construct specific inputs where part of the output $H$ is equal to a target value $\bar{H}$. To achieve such a partial preimage attack, we use truncated differentials built with the cancellation property, and we express the constraints needed on the state of the Feistel network in order to have the cancellation with probability one. We use degrees of freedom in the inputs of the compression function to satisfy those constraints. Then, we can compute some part of the output as a function of some of the remaining degrees of freedom, and try to invert the equation. The main idea is to obtain a simple equation that can be easily inverted using cancellations to limit the diffusion.

A partial preimage attack on the compression function allows to choose $k$ bits of the output for a cost of $2^{t}$ (with $t<k$ ), while the remaining $n-k$ bits are random. We can use such an attack on the compression function to target the hash function itself, in several scenarios.

Preimage Attacks. By repeating such an attack $2^{n-k}$ times, we can obtain a full preimage attack on the compression function, with complexity $2^{n+t-k}$. This preimage attack on the compression function can be used for a second preimage attack on the hash function with complexity $2^{n+(t-k) / 2}$ using a standard unbalanced meet-in-the middle [8]. Note that $2^{n+(t-k) / 2}<2^{n}$ if $t<k$.

Moreover, we point out that Lesamnta is built following the Matyas-MeyerOseas construction, i.e. the chaining value is used as a key, and the message enters the Feistel rounds. Since our partial preimage attack does not use degrees 
of freedom in the key (we only need the key to be known, not chosen), we can use a chaining value reached from the $I V$ as the key. We have to repeat the partial preimage attack with many different keys in order to build a full preimage, but we can use a first message block to randomize the key. This gives a second preimage attack on the hash function with complexity $2^{t+n-k}$.

Collision Attacks. The partial preimage attack can also be used to find collisions in the compression function. By generating $2^{(n-k) / 2}$ inputs where $k$ bits of the output are fixed to a common value, we expect a collision thanks to the birthday paradox. This collision attack on the compression function costs $2^{t+(n-k) / 2}$. If $t<k / 2$, this is more efficient than a generic birthday attack on the compression function.

If the compression function is built with the Matyas-Meyer-Oseas mode, like Lesamnta, this attack translates to a collision attack on the hash function with the same complexity. However, if the compression function follows the DaviesMeyer mode, like SHAvite-3, this does not translate to an attack on the hash function.

\subsection{Our Results}

The first candidate for the technique is the Lesamnta hash function. The best known generic attack against this structure is a 16-round attack by Mendel described in the submission document of Lesamnta 6]. Using a cancellation property, we extend this attack to a generic attacks on 22-round Lesamnta. The attack allows to fix one of the output words for an amortized cost of 1 , which gives collisions in time $2^{3 n / 8}$ and second preimages in time $2^{3 n / 4}$ for Lesamnta-n. Moreover, the preimage attack can be extended to 24 rounds using $2^{n / 4}$ memory. We follow with adaptations of the 24-round attacks without memory using specific properties of Lesamnta's round function.

The second target for our technique is the hash function SHAvite-3512. We show a 9-round attack using a cancellation property on the generalized Feistel structure of SHAvite-3512. The attack also works for the tweaked version of SHAvite- $3_{512}$, and allows fixing one out of the four output words. This allows a second preimage attack on 9 -round SHAvite- $3_{512}$ that takes about $2^{448}$ time. Note that this attack has recently been improved in a follow-up work [5]. First a new technique was used to add one extra round at the beginning, leading to attacks on 10 rounds of the compression function. Second, a pseudo-attack against the full SHAvite- $3_{512}$ is described, using additional degrees of freedom in the salt input. The follow-up work has been published first because of calendar issues, but it is heavily based on this work which was available as a preprint to the authors of [5]. Moreover, in this paper, we describe a more efficient way to find a suitable key for the attack, which improves the 10-round attack of [5].

In the full version of this paper, we also show some applications to block ciphers, with an integral attack on 21 rounds of the inner block cipher of Lesamnta, and a new truncated differential for $S_{M S} 4$. 
Table 1. Cancellation property on Lesamnta

On the left side, we have full diffusion after 9 rounds. On the right side, we use the cancellation property to control the diffusion of the differences.

\begin{tabular}{cccccc}
\hline$i$ & $S_{i}$ & $T_{i}$ & $U_{i}$ & $V_{i}$ & \\
\cline { 1 - 5 } 0 & $x$ & - & - & - & \\
1 & - & $x$ & - & - & \\
2 & - & - & $x$ & - & \\
3 & $y_{1}$ & - & - & $x$ & $x \rightarrow y_{1}$ \\
4 & $x$ & $y_{1}$ & - & - & \\
5 & - & $x$ & $y_{1}$ & - & \\
6 & $z$ & - & $x$ & $y_{1}$ & $y_{1} \rightarrow z$ \\
7 & $y^{\prime}$ & $z$ & - & $x$ & $x \rightarrow y_{2}, y^{\prime}=y_{1} \oplus y_{2}$ \\
8 & $x$ & $y^{\prime}$ & $z$ & - & \\
9 & $w$ & $x$ & $y^{\prime}$ & $z$ & $z \rightarrow w$ \\
\hline & & & &
\end{tabular}

\begin{tabular}{ccccc}
\hline$S_{i}$ & $T_{i}$ & $U_{i}$ & $V_{i}$ & \\
\cline { 1 - 4 }$x$ & - & - & - & \\
- & $x$ & - & - & \\
- & - & $x$ & - & \\
$y$ & - & - & $x$ & $\underline{x \rightarrow y}$ \\
$x$ & $y$ & - & - & \\
- & $x$ & $y$ & - & \\
$z$ & - & $x$ & $y$ & $y \rightarrow z$ \\
$\overline{-}$ & $z$ & - & $x$ & $\underline{x \rightarrow y}$ \\
$w$ & - & $z$ & - & \\
$w$ & $x$ & - & $z$ & $z \rightarrow w$ \\
\hline
\end{tabular}

The paper is organized as follows. Section 2 explains the basic idea of our cancellation attacks. Our results on Lesamnta are presented in Section 3, while application to SHAvite-3 $3_{512}$ is discussed in Section 4 . These results are summarized in Tables 9 and 10.

\section{The Cancellation Property}

In this paper we apply cancellation cryptanalysis to generalized Feistel schemes. The main idea of this technique is to impose constraints on the values of the state in order to limit the diffusion in the Feistel structure. When attacking a hash function, we have many degrees of freedom from the message and the chaining value, and it is important to find efficient ways to use those degrees of freedom.

Table 1 shows the diffusion of a single difference in Lesamnta. After 9 rounds, all the state words are active. However, we note that if the transitions $x \rightarrow y_{1}$ at rounds 3 and $x \rightarrow y_{2}$ at round 7 actually go to the same $y$, i.e. $y_{1}=y_{2}$, this limits the diffusion. In the ideal case, the round functions are all independent, and the probability of getting the same output difference is very small. However, in practice, the round functions are usually all derived from a single fixed permutation (or function). Therefore, if we add some constraints so that the input values of the fixed permutation at round 3 and 7 are the same, then we have the same output values, and therefore the same output difference with probability one. This is the cancellation property. A similar property can be used in SHAvite-3 512 .

Our attacks use an important property of the Feistel schemes of Lesamnta and SHAvite- $3_{512}$ : the diffusion is relatively slow. When a difference is introduced in the state, it takes several rounds to affect the full state and two different round functions can receive the same input difference $x$. Note that the slow diffusion of Lesamnta is the basis of a 16-round attack in [6] (recalled in Section 3.1), and 
the slow diffusion of SHAvite-3 ${ }_{512}$ gives a similar 8-round attack [4]. Our new attacks can be seen as extensions of those.

We now describe how to enforce conditions of the state so as to have this cancellation with probability 1 . Our attacks are independent of the round function, as long as all the round functions are derived from a single function as $F_{i}\left(X_{i}\right) \triangleq F\left(K_{i} \oplus X_{i}\right)$.

\subsection{Generic Properties of $F_{i}\left(X_{i}\right)=F\left(K_{i} \oplus X_{i}\right)$}

We assume that the round functions $F_{i}$ are built by applying a fixed permutation (or function) $F$ to $K_{i} \oplus X_{i}$, where $K_{i}$ is a round key and $X_{i}$ is the state input. This practice is common in many primitives like DES, SMS4, GOST, or Lesamnta.

This implies the followings, for all $i, j, k$ :

(i) $\exists c_{i, j}: \forall x, F_{i}\left(x \oplus c_{i, j}\right)=F_{j}(x)$.

(ii) $\forall \alpha, \#\left\{x: F_{i}(x) \oplus F_{j}(x)=\alpha\right\}$ is even.

(iii) $\bigoplus_{x} F_{k}\left(F_{i}(x) \oplus F_{j}(x)\right)=0$.

Property $(i)$ is the basis of our cancellation attack. We refer to it as the cancellation property. It states that if the inputs of two round functions are related by a specific fixed difference, then the outputs of both rounds are equal. The reminder of the paper is exploring this property.

Properties ( $(\mathrm{ii})$ and $(\mathrm{iii})$ can be used in an integral attack, as shown in the full version [3]. Note that Property $(\mathrm{ii})$ is a well known fact from differential cryptanalysis.

Proof. (i) Set $c_{i j}=K_{i} \oplus K_{j}$.

(ii) If $K_{i}=K_{j}$, then $\forall x, F_{i}(x) \oplus F_{j}(x)=0$. Otherwise, let $x$ be such that $F_{i}(x) \oplus F_{j}(x)=\alpha$. Then $F_{i}\left(x \oplus K_{i} \oplus K_{j}\right) \oplus F_{j}\left(x \oplus K_{i} \oplus K_{j}\right)=F_{j}(x) \oplus F_{i}(x)=$ $\alpha$. Therefore $x$ is in the set if and only if $x \oplus K_{i} \oplus K_{j}$ is in the set, and all the elements can be grouped in pairs.

(iii) Each term $F_{k}(\alpha)$ in the sum appears an even number of times, by $(i i)$.

\subsection{Using the Cancellation Property}

To better explain the cancellation property, we describe how to use it with the truncated differential of Table 1. In Table 2, we show the values of the registers during the computation of the truncated differential, starting at round 2 with $\left(S_{2}, T_{2}, U_{2}, V_{2}\right)=(a, b, c, d)$. To use the cancellation property, we want to make $S_{7}$ independent of $c$. Since we have $S_{7}=F_{6}\left(F_{3}(b) \oplus \underline{c)} \oplus F_{2} \underline{(c)} \oplus d\right.$, we can cancel the highlighted terms using property $(i)$. The dependency of $S_{7}$ on $c$ disappears if $F_{3}(b)=K_{2} \oplus K_{6}$, i.e. if $b=F_{3}^{-1}\left(K_{2} \oplus K_{6}\right)$ :

$$
S_{7}=F_{6}\left(F_{3}(b) \oplus c\right) \oplus F_{2}(c) \oplus d=F\left(K_{6} \oplus K_{2} \oplus K_{6} \oplus c\right) \oplus F\left(K_{2} \oplus c\right) \oplus d=d .
$$

Therefore, we can put any value $c$ in $U_{2}$, and it does not affect $S_{7}$ as long as we fix the value of $T_{2}$ to be $F^{-1}\left(K_{2} \oplus K_{6}\right) \oplus K_{3}$. Note that in a hash function, we 
Table 2. Values of the Registers for Five Rounds of Lesamnta

\begin{tabular}{|c|c|c|c|c|}
\hline$i$ & $S_{i}$ & $T_{i}$ & $U_{i}$ & $V_{i}$ \\
\hline 2 & $a$ & $b$ & $c$ & $d$ \\
\hline 3 & $F_{2}(c) \oplus d$ & $a$ & $b$ & $c$ \\
\hline 4 & $F_{3}(b) \oplus c$ & $F_{2}(c) \oplus d$ & $a$ & $b$ \\
\hline 5 & $F_{4}(a) \oplus b$ & $F_{3}(b) \oplus c$ & $F_{2}(c) \oplus d$ & $a$ \\
\hline 6 & $F_{5}\left(F_{2}(c) \oplus d\right) \oplus a$ & $F_{4}(a) \oplus b$ & $F_{3}(b) \oplus c$ & $F_{2}(c) \oplus d$ \\
\hline 7 & $F_{6}\left(F_{3}(b) \oplus \underline{c)} \oplus F_{2}(c) \oplus d\right.$ & $F_{5}\left(F_{2}(c) \oplus d\right) \oplus a$ & $F_{4}(a) \oplus b$ & $F_{3}(b) \oplus c$ \\
\hline
\end{tabular}

can compute $F^{-1}\left(K_{2} \oplus K_{6}\right) \oplus K_{3}$ since the keys are known to the adversary (or controlled by him), and we can choose to have this value in $T_{2}$.

This shows the three main requirements of our cancellation attacks:

- The generalized Feistel structures we study have a relatively slow diffusion. Therefore, the same difference can be used as the input difference of two different round functions.

- The round functions are built from a fixed permutation (or a fixed function), using a small round key. This differs from the ideal Luby-Rackoff case where all round functions are chosen independently at random.

- In a hash function setting the key is known to the adversary, and he can control some of the inner values.

Note that some of these requirements are not strictly necessary. For example, we show a 21-round integral attack on Lesamnta, without knowing the keys in the full version. Moreover, in Section 4 we show attacks on 9-round SHAvite-3 512 , where the round functions use more keying material.

\section{Application to Lesamnta}

Lesamnta is a hash function proposal by Hirose, Kuwakado, and Yoshida as a candidate in the SHA-3 competition 6]. It is based on a 32-round unbalanced Feistel scheme with four registers used in MMO mode. The key schedule is also based on a similar Feistel scheme. The round function can be written as:

$$
S_{i+1}=V_{i} \oplus F\left(U_{i} \oplus K_{i}\right) \quad T_{i+1}=S_{i} \quad U_{i+1}=T_{i} \quad V_{i+1}=U_{i}
$$

\subsection{Previous Results on Lesamnta}

The best known attack on Lesamnta is the self-similarity attack of [2]. Following this attack, the designers have tweaked Lesamnta by changing the round constants [10. In this paper we consider attacks that work with any round constants, and thus are applicable to the tweaked version as well. 
Several attacks on reduced-round Lesamnta are presented in the submission document [6]. A series of 16-round attacks for collisions and (second) preimage attacks are presented, all of which are based on a 16-round truncated differential with probability 1 .

In the next sections we show new attacks using the cancellation property. We first show some attacks that are generic in $F$, as long as the round functions are defined as $F_{i}\left(X_{i}\right)=F\left(K_{i} \oplus X_{i}\right)$, and then improved attacks using specific properties of the round functions of Lesamnta.

\subsection{Generic Attacks}

Our attacks are based on the differential of Table 3. which is an extension of the differential of Table 1, In this differential we use the cancellation property three times to control the diffusion. Note that we do not have to specify the values of $y$, $z, w, r$ and $t$. This specifies a truncated differential for Lesamnta: starting from a difference $(x,-,-,-)$, we reach a difference $\left(?, ?, ?, x_{1}\right)$ after 22 rounds. In order to use this truncated differential in our cancellation attack, we use two important properties: first, by adding constraints on the state, the truncated differential is followed with probability 1 ; second, the transition $x \rightarrow x_{1}$ is known because the key and values are known. Therefore, we can actually adjust the value of the last output word.

Table 3. Cancellation Property on 22 Rounds of Lesamnta

\begin{tabular}{ccccc}
\hline$i$ & $S_{i}$ & $T_{i}$ & $U_{i}$ & $V_{i}$ \\
\hline 0 & $x$ & - & - & - \\
1 & - & $x$ & - & - \\
2 & - & - & $x$ & - \\
3 & $y$ & - & - & $x$ \\
4 & $x$ & $y$ & - & - \\
5 & - & $x$ & $y$ & - \\
6 & $z$ & - & $x$ & $y$ \\
7 & - & $z$ & - & $x$ \\
8 & $x$ & - & $z$ & - \\
9 & $w$ & $x$ & - & $z$ \\
10 & $z$ & $w$ & $x$ & - \\
11 & $x_{1}$ & $z$ & $w$ & $x$ \\
\hline
\end{tabular}

\begin{tabular}{|c|c|c|c|c|c|c|}
\hline & 12 & $r$ & $x_{1}$ & $z$ & $w$ & $w \rightarrow x \oplus$ \\
\hline & 13 & - & $r$ & $x_{1}$ & $z$ & $z \rightarrow w$ \\
\hline & 14 & $?$ & 二 & $r$ & $x_{1}$ & \\
\hline & 15 & $x_{1}+t$ & $\bar{?}$ & - & $r$ & $\underline{r \rightarrow t}$ \\
\hline \multirow[t]{3}{*}{$x \rightarrow y$} & 16 & $r$ & $x_{1}+t$ & $\bar{?}$ & - & \\
\hline & 17 & $?$ & $r$ & $x_{1}+t$ & $\bar{?}$ & \\
\hline & 18 & $?$ & $?$ & $r$ & $x_{1}+t$ & \\
\hline$y \rightarrow z$ & 19 & $\underline{x_{1}}$ & $?$ & $?$ & $r$ & $\underline{r \rightarrow t}$ \\
\hline$x \rightarrow y$ & 20 & $\bar{?}$ & $\underline{x_{1}}$ & $?$ & $?$ & \\
\hline & 21 & $?$ & $\bar{?}$ & $\underline{x_{1}}$ & $?$ & \\
\hline$z \rightarrow w$ & 22 & $?$ & $?$ & $\bar{?}$ & $\underline{x_{1}}$ & \\
\hline$x \rightarrow x_{1}$ & $\mathrm{FF}$ & $?$ & $?$ & $?$ & $x_{1}$ & \\
\hline
\end{tabular}

In order to express the constraints that we need for the cancellation properties, we look at the values of the registers for this truncated differential. In Table 4 , we begin at round 2 with $\left(S_{2}, T_{2}, U_{2}, V_{2}\right)=(a, b, c, d)$, and we compute the state values up to round 19. This is an extension of the values computed in Table 2,

We can see that we have $S_{19}=F(c \oplus \alpha) \oplus \beta$, where $\alpha=K_{10} \oplus F_{7}\left(F_{4}(a) \oplus\right.$ b) $\oplus F_{3}(b)$ and $\beta=d$ provided that $(a, b, d)$ is the unique triplet satisfying the following cancellation conditions: 
Round 7: we have $F_{6}\left(F_{3}(b) \oplus \underline{c}\right) \oplus F_{2} \underline{(c)}$. They cancel if:
$F_{3}(b)=c_{2,6}=K_{2} \oplus K_{6}$
i.e. $b=F_{3}^{-1}\left(K_{2} \oplus K_{6}\right)$

Round 13: we have $F_{12}\left(F_{9}(d) \oplus \underline{\left.F_{5}\left(F_{2}(c) \oplus d\right) \oplus a\right)} \oplus F_{8} \underline{\left(F_{5}\left(F_{2}(c) \oplus d\right) \oplus a\right)}\right.$.

They cancel if:
$F_{9}(d)=c_{8,12}=K_{8} \oplus K_{12}$
i.e. $d=F_{9}^{-1}\left(K_{8} \oplus K_{12}\right)$

Round 19: we have $F_{18}\left(F_{15}\left(F_{4}(a) \oplus b\right) \oplus \underline{\left.S_{12}\right)} \oplus F_{14} \underline{\left(S_{12}\right)}\right.$. They cancel if:

$F_{15}\left(F_{4}(a) \oplus b\right)=c_{14,18}=K_{14} \oplus K_{18} \quad$ i.e. $a=F_{4}^{-1}\left(F_{15}^{-1}\left(K_{14} \oplus K_{18}\right) \oplus b\right)$

Note that $a, b, d$ and $\alpha, \beta$ are uniquely determined from the subkeys. Hence, one can set $S_{19}$ to any desired value $S_{19}^{*}$ by setting $c=F^{-1}\left(S_{19}^{*} \oplus \beta\right) \oplus \alpha$.

Table 4. Values of the Register for the 22-round Cancellation Property of Lesamnta. Steps -5 to -2 will be used for the 24-round attacks.

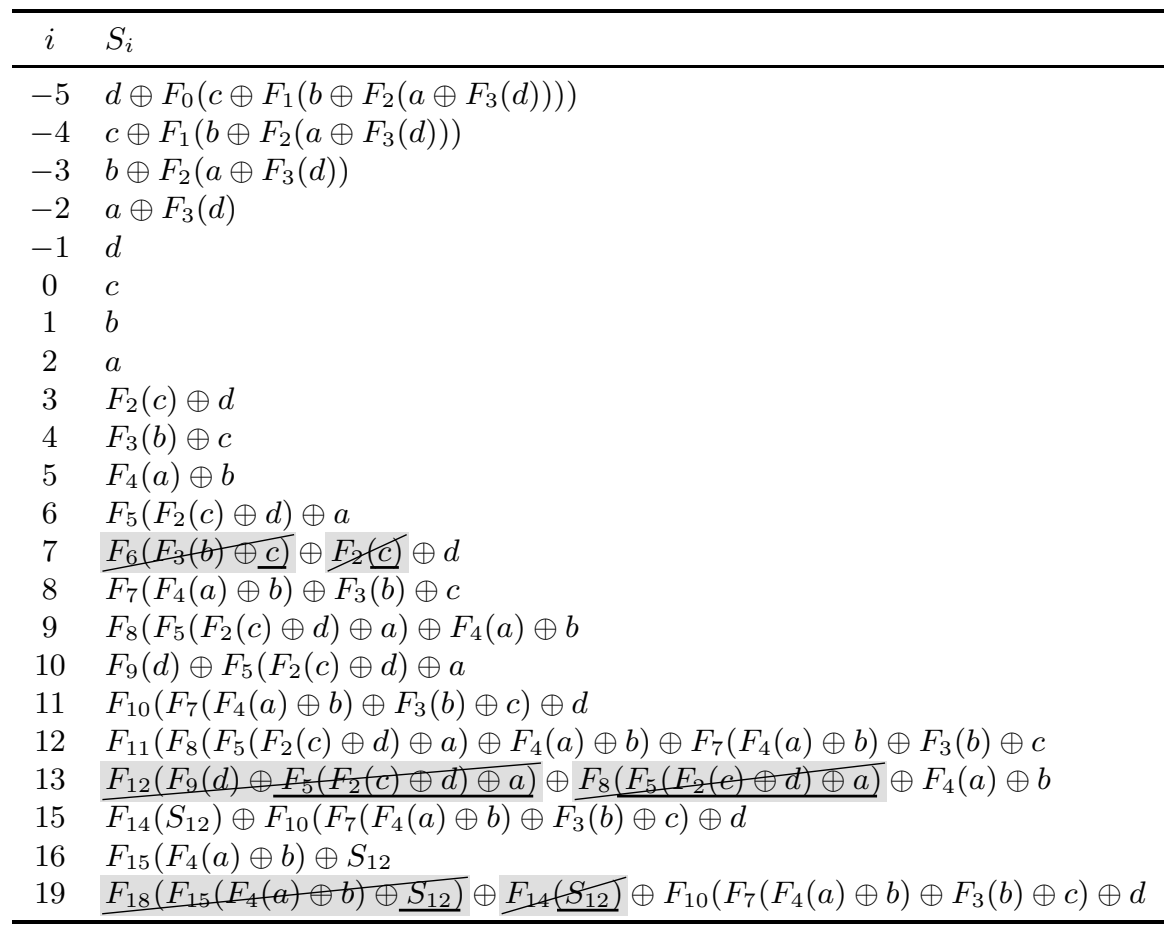

22-Round Attacks. The truncated differential of Table 3 can be used to attack 22-round Lesamnta. We start with the state at round $2\left(S_{2}, T_{2}, U_{2}, V_{2}\right)=$ $(a, b, c, d)$ satisfying the cancellation properties, and we can compute how the various states depend on $c$, as shown in Table 5. A dash (-) is used to denote a value that is independent of $c$. We know exactly how $c$ affects the last output word, and we can select $c$ in order to get a specific value at the output. Suppose we are given a set of subkeys, and a target value $\bar{H}$ for the fourth output word. Then the attack proceeds as follows: 
Table 5. Collision and Preimage Characteristic for the 22-Round Attack

\begin{tabular}{ccccc}
\hline$i$ & $S_{i}$ & $T_{i}$ & $U_{i}$ & $V_{i}$ \\
\hline 0 & $c$ & - & - & $\eta$ \\
1 & - & $c$ & - & - \\
2 & - & - & $c$ & - \\
\hline $2-19$ & & Repeated Cancellation & Property: Table 4 \\
\hline 19 & $F(c \oplus \alpha) \oplus \beta$ & $?$ & $?$ & $?$ \\
20 & $?$ & $F(c \oplus \alpha) \oplus \beta$ & $?$ & $?$ \\
21 & $?$ & $?$ & $F(c \oplus \alpha) \oplus \beta$ & $?$ \\
22 & $?$ & $?$ & $?$ & $F(c \oplus \alpha) \oplus \beta$ \\
\hline FF & $?$ & $?$ & $?$ & $\eta \oplus F(c \oplus \alpha) \oplus \beta$ \\
\hline
\end{tabular}

$\eta, \alpha$ and $\beta$ can be computed from $a, b, d$ and the key:

$\eta=b \oplus F_{0}\left(a \oplus F_{3}(d)\right), \alpha=K_{11} \oplus F_{8}\left(F_{5}(a) \oplus b\right) \oplus F_{4}(b), \beta=d$.

1. Set $a, b$, and $d$ to the values that allow the cancellation property. Then we have $V_{0} \oplus V_{22}=\eta \oplus F(c \oplus \alpha) \oplus \beta$, as shown in Table 5.

2. Compute $c$ as $F^{-1}(\bar{H} \oplus \eta \oplus \beta) \oplus \alpha$.

3. This sets the state at round 2: $\left(S_{2}, T_{2}, U_{2}, V_{2}\right) \triangleq(a, b, c, d)$.

With this state, we have $V_{0} \oplus V_{22}=\bar{H}$.

4. Compute the round function backwards up to round 0 , to get the input.

This costs less than one compression function call, and does not require any memory.

For a given chaining value (i.e. a set of subkeys), this algorithm can only output one message. To build a full preimage attack or a collision attack on the compression function, this has to be repeated with random chaining values. Since the attack works for any chaining value, we can build attacks on the hash function using a prefix block to randomize the chaining value. This gives a collision attack with complexity $2^{96}$ ( $2^{192}$ for Lesamnta-512), and a second-preimage attack with complexity $2^{192}\left(2^{384}\right.$ for Lesamnta-512).

24-Round Attacks. We can add two rounds at the beginning of the truncated differential at the cost of some memory. The resulting 24-round differential is given in Table6. The output word we try to control is equal to $F(c \oplus \gamma) \oplus F(c \oplus \alpha)$, for some constants $\alpha$, and $\gamma$ that depend on the chaining value (note that $\beta=\lambda$ in Table 6). We define a family of functions $h_{\mu}(x)=F(x) \oplus F(x \oplus \mu)$, and for a given target value $\bar{H}$, we tabulate $\varphi_{\bar{H}}(\mu)=h_{\mu}^{-1}(\bar{H})$. For each $\mu, \varphi_{\bar{H}}(\mu)$ is a possibly empty set, but the average size is one (the non-empty values form a partition of the input space). In the special case where $\bar{H}=0, \varphi_{0}(\mu)$ is empty for all $\mu \neq 0$, and $\varphi_{0}(0)$ is the full space. 
Table 6. Collision and Preimage Path for the 24-round Attack

\begin{tabular}{ccccc}
\hline$i$ & $S_{i}$ & $T_{i}$ & $U_{i}$ & $V_{i}$ \\
\hline 0 & - & - & $c \oplus \gamma$ & $F(c \oplus \gamma) \oplus \lambda$ \\
1 & - & - & - & $c \oplus \gamma$ \\
2 & $c$ & - & - & - \\
3 & - & $c$ & - & - \\
4 & - & - & $c$ & - \\
\hline
\end{tabular}

\begin{tabular}{ccccc}
\hline $4-21$ & \multicolumn{4}{c}{ Repeated Cancellation Property: Table 4} \\
\hline 21 & $F(c \oplus \alpha) \oplus \beta$ & $?$ & $?$ & $?$ \\
22 & $?$ & $F(c \oplus \alpha) \oplus \beta$ & $?$ & $?$ \\
23 & $?$ & $?$ & $F(c \oplus \alpha) \oplus \beta$ & $?$ \\
24 & $?$ & $?$ & $?$ & $F(c \oplus \alpha) \oplus \beta$ \\
\hline
\end{tabular}

$\alpha, \beta, \gamma$ and $\lambda$ can be computed from $a, b, d$ and the key by:

$\alpha=K_{13} \oplus F_{10}\left(F_{7}(a) \oplus b\right) \oplus F_{6}(b), \beta=d$ and

$\gamma=F_{1}\left(b \oplus F_{2}\left(a \oplus F_{3}(d)\right)\right), \lambda=d$

We store $\varphi_{\bar{H}}$ in a table of size $2^{n / 4}$, and we can compute it in time $2^{n / 4}$ by looking for values such that $F(x) \oplus F(y)=\bar{H}$ (this gives $\varphi_{\bar{H}}(x \oplus y)=x$ ). Using this table, we are able to choose one output word just like in the 22-round attack.

We start with a state $\left(S_{4}, T_{4}, U_{4}, V_{4}\right)=(a, b, c, d)$ such that $a, b, d$ satisfy the cancellation conditions, and we compute $\alpha, \beta, \gamma, \lambda$. If we use $c=u \oplus \alpha$, where $u \in \varphi_{\bar{H}}(\alpha \oplus \gamma)=h_{\alpha \oplus \gamma}^{-1}(\bar{H})$, we have:

$$
V_{0} \oplus V_{24}=F(c \oplus \gamma) \oplus F(c \oplus \alpha)=F(u \oplus \alpha \oplus \gamma) \oplus F(u)=h_{\alpha \oplus \gamma}(u)=\bar{H}
$$

On average this costs one compression function evaluation to find a $n / 4$-bit partial preimage. If the target value is 0 , this only succeeds if $\alpha \oplus \gamma=0$, but in this case it gives $2^{n / 4}$ solutions. This gives a preimage attack with complexity $2^{3 n / 4}$ using $2^{n / 4}$ memory.

Note that it is possible to make a time-memory trade-off with complexity $2^{n-k}$ using $2^{k}$ memory for $k<n / 4$.

\subsection{Dedicated 24-Round Attacks on Lesamnta}

We now describe how to use specific properties of the round functions of Lesamnta to remove the memory requirement of our 24-round attacks.

Slow Diffusion in $\boldsymbol{F}_{\mathbf{2 5 6}}$. The AES-like round function of Lesamnta-256 achieves full diffusion of the values after its four rounds, but some linear combinations of the output are not affected. Starting from a single active diagonal, we have:

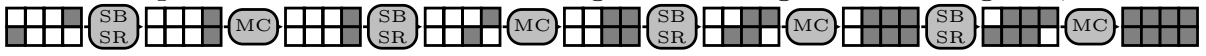
All the output bytes are active, but there are some linear relations between them. More precisely, the inverse MixColumns operation leads to a difference with two inactive bytes. 
This gives two linear subspaces $\Gamma$ and $\Lambda$ for which $x \oplus x^{\prime} \in \Gamma \Rightarrow F(x) \oplus F\left(x^{\prime}\right) \in$ $\Lambda$. The subspaces $\Gamma$ and $\Lambda$ have dimensions of 16 and 48 , respectively.

Collision and Second Preimage Attacks on Lesamnta-256. Using this property, we can choose 16 linear relations of the output of the family of function $h_{\mu}$, or equivalently, choose 16 linear relations of the output of the compression function.

Let $\bar{\Lambda}$ be a supplementary subspace of $\Lambda$. Any 64 -bit value $x$ can be written as $x=x_{\Lambda}+x_{\bar{\Lambda}}$, where $x_{\Lambda} \in \Lambda$ and $x_{\bar{\Lambda}} \in \bar{\Lambda}$. We can find values $x$ such that $h_{\mu}(x)_{\bar{\Lambda}}=\bar{H}_{\bar{\Lambda}}$ for an amortized cost of one, without memory:

1. Compute $h_{\mu}(u)$ for random $u$ 's until $h_{\mu}(u)_{\bar{\Lambda}}=\bar{H}_{\bar{\Lambda}}$

2. Far all $v$ in $\Gamma$, we have $h_{\mu}(u+v)_{\bar{\Lambda}}=\bar{H}_{\bar{\Lambda}}$

This gives $2^{16}$ messages with 16 chosen relations for a cost of $2^{16}$. It allows a second-preimage attack on 24-round Lesamnta-256 with complexity $2^{240}$, and a collision attack with complexity $2^{120}$, both memoryless.

Symmetries in $\boldsymbol{F}_{\mathbf{2 5 6}}$ and $\boldsymbol{F}_{\mathbf{5 1 2}}$. The AES round function has strong symmetry properties, as studied in 9 . The round function $F$ of Lesamnta is heavily inspired by the AES round, and has similar symmetry properties. More specifically, if an AES state is such that the left half is equal to the right half, then this property still holds after any number of SubBytes, ShiftRows, and MixColumns operations.

When we consider the $F$ functions of Lesamnta, we have that: if $x \oplus K_{i}$ is symmetric, then $F_{i}(x)=F\left(x \oplus K_{i}\right)$ is also symmetric.

Collision Attacks on Lesamnta-256 and Lesamnta-512. This property can be used for an improved collision attack. As seen earlier we have $V_{0} \oplus V_{24}=F(c \oplus$ $\gamma) \oplus F(c \oplus \alpha)$. In order to use the symmetry property, we first select random chaining values, and we compute the value of $\alpha$ and $\gamma$ until $\alpha \oplus \gamma$ is symmetric. Then, if we select $c$ such that $c \oplus \gamma$ is symmetric, we have that $V_{0} \oplus V_{24}$ is symmetric.

This leads to a collision attack with complexity $2^{112}$ for Lesamnta-256, and $2^{224}$ for Lesamnta-512.

\section{Application to $S H A v i t e-3_{512}$}

SHAvite-3 is a hash function designed by Biham and Dunkelman for the SHA-3 competition [1]. It is based on a generalized Feistel construction with an AESbased round function, used in Davies-Meyer mode. In this section we study SHAvite-3512, the version of SHAvite-3 designed for output size of 257 to 512 bits. The cancellation property can not be used on SHAvite- $3_{256}$ because the Feistel structure is different and has a faster diffusion. We describe an attack on the SHAvite- ${ }_{512}$ hash function reduced to 9 rounds out of 14 . An earlier variant of our attack was later extended in [5] to a 10-round attack. We note that our improved 9-round attack can be used to offer an improved 10-round attack. 


\subsection{A Short Description of SHAvite- $3_{512}$}

The compression function of SHAvite- $3_{512}$ accepts a chaining value of 512 bits, a message block of 1024 bits, a salt of 512 bits, and a bit counter of 128 bits. As this is a Davies-Meyer construction, the message block, the salt, and the bit counter enter the key schedule algorithm of the underlying block cipher. The key schedule algorithm transforms them into 112 subkeys of 128 bits each. The chaining value is then divided into four 128-bit words, and at each round two words enter the nonlinear round functions and affect the other two:

$$
S_{i+1}=V_{i} \quad T_{i+1}=S_{i} \oplus F_{i}^{\prime}\left(T_{i}\right) \quad U_{i+1}=T_{i} \quad V_{i+1}=U_{i} \oplus F_{i}\left(V_{i}\right)
$$

The nonlinear function $F$ and $F^{\prime}$ are composed of four full rounds of AES, with 4 subkeys from the message expansion:

$$
\begin{aligned}
& F_{i}(x)=P\left(k_{0, i}^{3} \oplus P\left(k_{0, i}^{2} \oplus P\left(k_{0, i}^{1} \oplus P\left(k_{0, i}^{0} \oplus x\right)\right)\right)\right) \\
& F_{i}^{\prime}(x)=P\left(k_{1, i}^{3} \oplus P\left(k_{1, i}^{2} \oplus P\left(k_{1, i}^{1} \oplus P\left(k_{1, i}^{0} \oplus x\right)\right)\right)\right)
\end{aligned}
$$

where $P$ is one AES round (without the AddRoundKey operation).

In this section we use an alternative description of SHAvite-3 ${ }_{512}$ with only two variables per round. We have

$$
S_{i}=Y_{i-1} \quad T_{i}=X_{i} \quad U_{i}=X_{i-1} \quad V_{i}=Y_{i}
$$

The message expansion generates an array $r k[\cdot]$ of 44832 -bit words by alternating linear steps and AES rounds:

Using the counter: the counter is used at 4 specific positions.

In order to simplify the description, we define a new table holding the preprocessed counter:

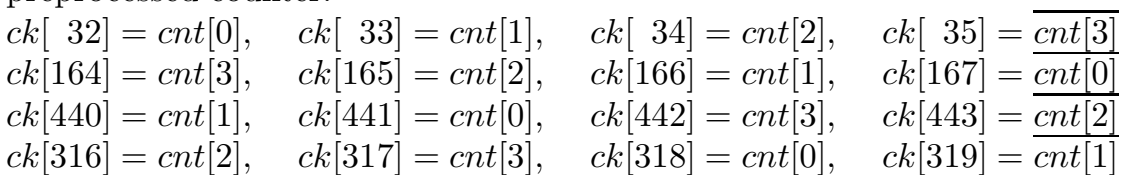

For all the other values, $c k[i]=0$.

AES rounds: for $i \in\{0,64,128,192,256,320,384\}+\{0,4,8,12,16,20,24,28\}$ : $t k[(i, i+1, i+2, i+3)]=\operatorname{AESR}(r k[(i+1, i+2, i+3, i)] \oplus \operatorname{salt}[(i, i+1, i+$ $2, i+3) \bmod 16])$

Linear Step 1: for $i \in\{32,96,160,224,288,352,416\}+\{0, \ldots, 31\}$ : $r k[i]=t k[i-32] \oplus r k[i-4] \oplus c k[i]$

Linear Step 2: for $i \in\{64,128,192,256,320,384\}+\{0, \ldots, 31\}$ : $r k[i]=r k[i-32] \oplus r k[i-7]$ 
Table 7. Cancellation Property on 9 Rounds of SHAvite-3512

\begin{tabular}{cccccl}
\hline$i$ & $S_{i}$ & $T_{i}$ & $U_{i}$ & $V_{i}$ & \\
\cline { 1 - 4 } 0 & $?$ & $x_{2}$ & $?$ & $x$ & \\
1 & $x$ & - & $x_{2}$ & $x_{1}$ & \\
2 & $x_{1}$ & $x$ & - & - & $x_{1} \rightarrow x_{2}$ \\
3 & - & - & $x$ & - & $x \rightarrow x_{1}$ \\
4 & - & - & - & $x$ & \\
5 & $x$ & - & - & $y$ & $x \rightarrow y$ \\
6 & $y$ & $x$ & - & $z$ & $y \rightarrow z$ \\
7 & $z$ & - & $x$ & $w$ & $\underline{x \rightarrow y} z \rightarrow w$ \\
8 & $w$ & $z$ & - & $?$ & \\
9 & $?$ & - & $z$ & $?$ & \\
\cline { 1 - 4 } & $?$ & $x_{2}$ & $?$ & $?$ & \\
\hline
\end{tabular}

Table 8. Values of the Registers for the 9-round Cancellation Property of SHAvite- $3_{512}$

\begin{tabular}{lll}
\hline$i$ & $X_{i}$ & $Y_{i}$ \\
\hline 0 & $b \oplus F_{3}(c) \oplus F_{1}^{\prime}\left(c \oplus F_{2}\left(d \oplus F_{3}^{\prime}(a)\right)\right)$ & $d \oplus F_{3}^{\prime}(a) \oplus F_{1}\left(a \oplus F_{2}^{\prime}\left(b \oplus F_{3}(c)\right)\right)$ \\
1 & $a \oplus F_{2}^{\prime}\left(b \oplus F_{3}(c)\right)$ & $c \oplus F_{2}\left(d \oplus F_{3}^{\prime}(a)\right)$ \\
2 & $d \oplus F_{3}^{\prime}(a)$ & $b \oplus F_{3}(c)$ \\
3 & $c$ & $a$ \\
4 & $b$ & $d$ \\
5 & $a \oplus F_{4}(b)$ & $c \oplus F_{4}^{\prime}(d)$ \\
6 & $d \oplus F_{5}\left(a \oplus F_{4}(b)\right)$ & $b \oplus F_{5}^{\prime}\left(c \oplus F_{4}^{\prime}(d)\right)$ \\
7 & $c \oplus F_{4}^{\prime}(d) \oplus F_{6}\left(d \oplus F_{5}\left(a \oplus F_{4}(b)\right)\right)$ & $a \oplus F_{4}(b) \oplus F_{6}^{\prime}\left(b \oplus F_{5}^{\prime}\left(c \oplus F_{4}^{\prime}(d)\right)\right)$ \\
8 & $b \oplus F_{5}^{\prime}\left(c \oplus \overline{\left.F_{4}^{\prime}(d)\right) \oplus F_{7}(c)}\right.$ & $?$ \\
9 & $a \oplus F_{4}(b) \oplus \underline{F_{6}^{\prime}\left(b \oplus F_{5}^{\prime}\left(c \oplus F_{4}^{\prime}(d)\right)\right)} \oplus \underline{F_{8}\left(b \oplus F_{5}^{\prime}\left(c \oplus F_{4}^{\prime}(d)\right) \oplus F_{7}(c)\right)}$ \\
\hline
\end{tabular}

\subsection{Cancellation Attacks on SHAvite- $_{512}$}

The cancellation path is described in Table 7 . We use the cancellation property twice to control the diffusion. Note that we do not have to specify the values of $y, z$, and $w$. Like in the Lesamnta attack, this path is a truncated differential, and we use constraints on the state to enforce that it is followed. Moreover, the transitions $x \rightarrow x_{1}$ and $x_{1} \rightarrow x_{2}$ are known because the key is known.

Note that the round functions of SHAvite- $3_{512}$ are not defined as $F(k, x)=$ $P(k \oplus x)$ for a fixed permutation $P$. Instead, each function takes 4 keys and it is defined as:

$$
F\left(k_{i}^{0}, k_{i}^{1}, k_{i}^{2}, k_{i}^{3}, x\right)=P\left(k_{i}^{3} \oplus P\left(k_{i}^{2} \oplus P\left(k_{i}^{1} \oplus P\left(k_{i}^{0} \oplus x\right)\right)\right)\right)
$$

where $P$ is one AES round. In order to apply the cancellation property to SHAvite- $3_{512}$, we need that the subkeys $k^{1}, k^{2}, k^{3}$ of two functions be equal, 
so that $F_{i}(x)$ collapses to $P^{\prime}\left(k_{i}^{0} \oplus x\right)$ and $F_{j}$ to $P^{\prime}\left(k_{j}^{0} \oplus x\right)$, where $P^{\prime}(x) \triangleq$ $P\left(k_{i}^{3} \oplus P\left(k_{i}^{2} \oplus P\left(k_{i}^{1} \oplus P(x)\right)\right)\right)=P\left(k_{j}^{3} \oplus P\left(k_{j}^{2} \oplus P\left(k_{j}^{1} \oplus P(x)\right)\right)\right)$.

In order to express the constraints needed for the cancellation properties, we look at the values of the registers for this truncated differential. In Table 8, we begin at round 4 with $\left(S_{4}, T_{4}, U_{4}, V_{4}\right)=\left(Y_{3}, X_{4}, X_{3}, Y_{4}\right)=(a, b, c, d)$, and we compute up to round 9 .

We have a cancellation property on 9 rounds under the following conditions:

Round 7. We have $\left.F_{4}^{\prime} \underline{(d)} \oplus F_{6} \underline{(d \oplus} \oplus F_{5}\left(a \oplus F_{4}(b)\right)\right)$. They cancel if: $F_{5}\left(a \oplus F_{4}(b)\right)=k_{1.4}^{0} \oplus k_{0.6}^{0}$ and $\left(k_{1.4}^{1}, k_{1,4}^{2}, k_{1,4}^{3}\right)=\left(k_{0.6}^{1}, k_{0.6}^{2}, k_{0.6}^{3}\right)$.

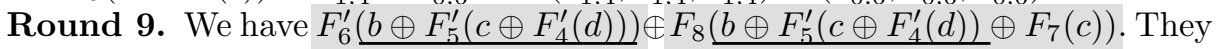
cancel if:

$F_{7}(c)=k_{1,6}^{0} \oplus k_{0,8}^{0}$ and $\left(k_{1,6}^{1}, k_{1,6}^{2}, k_{1,6}^{3}\right)=\left(k_{0,8}^{1}, k_{0,8}^{2}, k_{0,8}^{3}\right)$.

Therefore, the truncated differential is followed if:

$$
\begin{aligned}
F_{5}\left(a \oplus F_{4}(b)\right) & =k_{1,4}^{0} \oplus k_{0,6}^{0} & F_{7}(c) & =k_{1,6}^{0} \oplus k_{0,8}^{0} \\
\left(k_{1,4}^{1}, k_{1,4}^{2}, k_{1,4}^{3}\right) & =\left(k_{0,6}^{1}, k_{0,6}^{2}, k_{0,6}^{3}\right) & \left(k_{1,6}^{1}, k_{1,6}^{2}, k_{1,6}^{3}\right) & =\left(k_{0,8}^{1}, k_{0,8}^{2}, k_{0,8}^{3}\right)
\end{aligned}
$$

The constraints for the cancellation at round 7 are easy to satisfy and allow a 7-round attack on SHAvite-3512. However, for a 9-round attack we have more constraints on the subkeys, and this requires special attention.

\subsection{Dealing with the Key Expansion}

Let us outline an algorithm to find a suitable message (recall that SHAvite-3 ${ }_{512}$ is used in a Davies-Meyer mode) for a given salt and counter value. We have to solve a system involving linear and non-linear equations, and we use the fact that the system is almost triangular. We note that it might be possible to improve our results using the technique of Khovratovich, Biryukov and Nikolic [7] to find a good message efficiently.

For the cancellation attack on 9-round SHAvite-3 ${ }_{512}$, we need to satisfy a 768-bit condition on the subkeys, i.e.:

$$
\left(k_{1,4}^{1}, k_{1,4}^{2}, k_{1,4}^{3}\right)=\left(k_{0,6}^{1}, k_{0,6}^{2}, k_{0,6}^{3}\right) \quad\left(k_{1,6}^{1}, k_{1,6}^{2}, k_{1,6}^{3}\right)=\left(k_{0,8}^{1}, k_{0,8}^{2}, k_{0,8}^{3}\right)
$$

Or in $r k[\cdot]$ terms:

$$
r k[148, \ldots, 159]=r k[196, \ldots, 207] \quad r k[212, \ldots, 223]=r k[260, \ldots, 271]
$$

We are actually trying to solve a system of equation with:

- 224 variables: $t k[128 . .159], t k[192 . .223]$ and $r k[128 . .287]$

- 192 equations from the key schedule (64 non-linear and 128 linear)

-24 constraints

Therefore we have 8 degrees of freedom. The relations between the variables are shown in Figure 2, while the full key expansion of SHAvite-3 $_{512}$ is described in Appendix. 
$r k\left[\begin{array}{llllllllllllllllll}128 & \ldots & 131,132 & \ldots & 135,136 & \ldots & 139,140 & \ldots & 143,144 & \ldots & 147,148 & \ldots & 151,152 & \ldots & 155,156 & \ldots & 159\end{array}\right.$

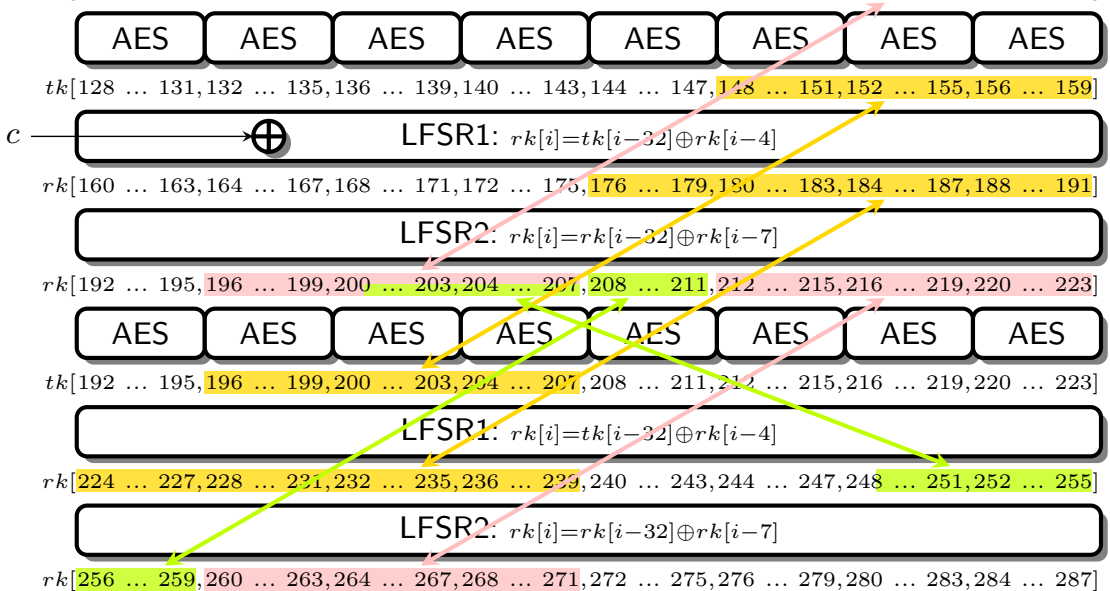

Fig. 2. Constraints in the Key Expansion of SHAvite-3512

Initial constraints in pink, constraints from steps 1 to 3 in yellow, constraints from step 4 in green

Propagation of the Constraints. First, we propagate the constraints and deduce new equalities between the variables. Figure 2 shows the initial constraints and the propagated constraints.

1. The non-linear equations of the key-schedule give:

$$
\begin{aligned}
& t k[156 . .159]=\operatorname{AESR}((r k[157,158,159,156]) \oplus(\operatorname{salt}[12 . .15])) \\
& t k[204 . .207]=\operatorname{AESR}((r k[205,206,207,204]) \oplus(\operatorname{salt}[12 . .15]))
\end{aligned}
$$

since $r k[156 . .159]=r k[204 . .207]$, we know that $t k[156 . .159]=t k[204 . .207]$. Similarly, we get $t k[148 . .159]=t k[196 . .207]$

2. From the key expansion, we have $r k[191]=r k[223] \oplus r k[216]$, and $r k[239]=$ $r k[271] \oplus r k[264]$. Since we have the constraints $r k[223]=r k[271]$ and $r k[216]=r k[264]$, we can deduce that $r k[191]=r k[239]$ Similarly, we get $r k[187 . .191]=r k[235 . .239]$.

3 . From the linear part of the expansion, we have $r k[186]=r k[190] \oplus t k[158]$ and $r k[234]=r k[238] \oplus t k[206]$. We have seen that $r k[190]=r k[238]$ at step 2 and $t k[158]=t k[206]$ at step 1, therefore $r k[186]=r k[234]$ Similarly, we get $r k[176 . .186]=r k[224 . .234]$.

4. Again, from the linear part of the key expansion, we have $r k[211]=r k[218] \oplus$ $r k[186]$ and $r k[259]=r k[266] \oplus r k[234]$. We have seen that $r k[186]=r k[234]$ at step 3 and we have $r k[218]=r k[266]$ as a constraint, thus $r k[211]=$ $r k[259]$ Similarly, we obtain $r k[201 . .211]=r k[249 . .259]$ Note that we have $r k[201 . .207]=r k[153 . .159]$ as a constraint, so we must have $r k[249 . .255]=$ $r k[153 . .159]$. 
Finding a Solution. To find a solution to the system, we use a guess and determine technique. We guess 11 state variables, and we show how to compute the rest of the state and check for consistency. Since we have only 8 degrees of freedom, we expect the random initial choice to be valid once out of $2^{32 \times 3}=2^{96}$ times. This gives a complexity of $2^{96}$ to find a good message.

- Choose random values for $r k[200], r k[204 . .207], r k[215 . .216], r k[220 . .223]$

- Compute $t k[220 . .223]$ from $r k[220 . .223]$

- Compute $r k[248 . .251]$ from $t k[220 . .223]$ and $r k[252 . .255]$ (=rk[204..207])

- Deduce $r k[201 . .203]=r k[249 . .251]$, so $r k[200 . .207]$ is known

- Compute $t k[152 . .159]$ from $r k[152 . .159]$ (= $r k[200 . .207])$

- Compute $r k[190 . .191]$ from $r k[215 . .216]$ and $r k[222 . .223]$

- Compute $r k[186 . .187]$ from $r k[190 . .191]$ and $r k[158 . .159]$

- Compute $r k[182 . .183]$ from $r k[186 . .187]$ and $r k[154 . .155]$

- Compute $r k[214]$ from $r k[207]$ and $r k[182]$

- Compute $r k[189]$ from $r k[214]$ and $r k[219] ;$ then $r k[185]$ and $r k[181]$

- Compute $r k[213]$ from $r k[206]$ and $r k[181]$

- Compute $r k[188]$ from $r k[213]$ and $r k[220]$, then $r k[184]$ and $r k[180]$

- Compute $r k[212]$ from $r k[205]$ and $r k[180]$

- Compute $r k[219]$ from $r k[212]$ and $r k[187]$

- Compute $r k[208,209]$ from $r k[215,216]$ and $r k[183,184]$

- We have $t k[216 . .219]=\operatorname{AESR}((r k[216 . .219]))$ with a known key. Since $r k[216]$ and $r k[219]$ are known, we know that $t k[216 . .219]$ is a linear subspace of dimension 64 over $\mathbb{F}_{2}$.

- Similarly, $t k[208 . .211]$ is in a linear subspace of dimension $64(r k[208]$ and $r k[209]$ are known).

- Moreover, there are linear relations between $t k[216 . .219]$ and $t k[208 . .211]$ : we can compute $r k[240 . .243]$ from $t k[208 . .211]$ and $r k[236 . .239] ; r k[244 . .247]$ from $r k[240 . .243]$ and $t k[212 . .215] ; t k[216 . .219]$ from $r k$ [244..247] and $r k[248 . .251]$.

- On average, we expect one solution for $t k[216 . .219]$ and $t k[208 . .211]$.

- At this point we have computed the values of $r k[200 . .223]$. We can compute $t k[200.223]$ and $r k[228 . .255]$.

- Compute $r k[176 . .179]$ from $r k[201 . .204]$ and $r k[208 . .211]$

- Since $r k[224 . .227]=r k[176 . .179]$, we have a full state $r k[224 . .255]$. We can check consistency of the initial guess.

\subsection{9-Round Attacks}

The cancellation property allows to find a key/message pair with a given value on the last 128 bits. The attack is the following: first find a message that fulfills the conditions on the subkeys, and set $a, b$ and $c$ at round 4 satisfying the cancellation conditions (C0). Then the second output word is:

$$
T_{9} \oplus T_{0}=X_{9} \oplus X_{0}=a \oplus F_{4}(b) \oplus b \oplus F_{3}(c) \oplus F_{1}^{\prime}\left(c \oplus F_{2}\left(d \oplus F_{3}^{\prime}(a)\right)\right)
$$


Table 9. Summary of the Attacks on the Lesamnta Hash Function

\begin{tabular}{|c|c|c|c|c|c|c|c|}
\hline & \multirow{2}{*}{\multicolumn{2}{|c|}{ Attack }} & \multirow[b]{2}{*}{ Rnds } & \multicolumn{2}{|c|}{ Lesamnta-256 } & \multicolumn{2}{|c|}{ Lesamnta-512 } \\
\hline & & & & Time & Mem. & Time & Mem. \\
\hline \multirow[t]{6}{*}{ Generic } & Collision & 6 & 16 & $2^{97}$ & - & $2^{193}$ & - \\
\hline & $2^{\text {nd }}$ Preimage & 6 & 16 & $2^{193}$ & - & $2^{385}$ & - \\
\hline & Collision & (Sect. 3.2) & 22 & $2^{96}$ & - & $2^{192}$ & - \\
\hline & $2^{\text {nd }}$ Preimage & (Sect. 3.2 ) & 22 & $2^{192}$ & - & $2^{384}$ & - \\
\hline & Collision & (Sect. 3.2 ) & 24 & $2^{96}$ & $2^{64}$ & $2^{192}$ & $2^{128}$ \\
\hline & $2^{\text {nd }}$ Preimage & (Sect. $\overrightarrow{3.2}$ & 24 & $2^{192}$ & $2^{64}$ & $2^{384}$ & $2^{128}$ \\
\hline \multirow[t]{2}{*}{ Specific } & Collision & (Sect. 3.3) & 24 & $2^{112}$ & - & $2^{224}$ & - \\
\hline & $2^{\text {nd }}$ Preimage & (Sect. $\overrightarrow{3.3}$ ) & 24 & $2^{240}$ & - & \multicolumn{2}{|c|}{$N / A$} \\
\hline
\end{tabular}

If we set

$$
d=F_{2}^{-1}\left(F_{1}^{\prime-1}\left(\bar{H} \oplus a \oplus F_{4}(b) \oplus b \oplus F_{3}(c)\right) \oplus c\right) \oplus F_{3}^{\prime}(a)
$$

we have $X_{9} \oplus X_{0}=\bar{H}$. Each key (message) can be used with $2^{128}$ different $a, b, c$, and the cost of finding a suitable key is $2^{96}$. Hence, the amortized cost for finding a 128-bit partial preimage is one compression function evaluation. The cost of finding a full preimage for the compression function is $2^{384}$.

Second Preimage Attack on the Hash Function. We can use the preimage attack on the compression function to build a second preimage attack on the hash function reduced to 9 rounds. Using a generic unbalanced meet-in-the-middle attack the complexity is about $2^{448}$ compression function evaluations and $2^{64}$ memory. Note that we cannot find preimages for the hash function because we cannot find correctly padded message blocks.

Table 10. Summary of the Attacks on SHAvite- $3_{512}$

\begin{tabular}{|c|c|c|c|c|c|c|}
\hline \multirow{2}{*}{\multicolumn{2}{|c|}{ Attack }} & \multirow[b]{2}{*}{ Rnds } & \multicolumn{2}{|c|}{ Comp. Fun. } & \multicolumn{2}{|c|}{ Hash Fun. } \\
\hline & & & Time & Mem. & Time & Mem. \\
\hline $2^{\text {nd }}$ Preimage & 4 & 8 & $2^{384}$ & - & $2^{448}$ & $2^{64}$ \\
\hline $2^{\text {nd }}$ Preimage & (Sect. 4.4) & 9 & $2^{384}$ & - & $2^{448}$ & $2^{64}$ \\
\hline $2^{\text {nd }}$ Preimage & (extension of this work) 5 & 10 & $2^{480}$ & - & $2^{496}$ & $2^{16}$ \\
\hline $2^{\text {nd }}$ Preimage & (improving [5] w/ Sect. 4.3] & 10 & $2^{448}$ & - & $2^{480}$ & $2^{32}$ \\
\hline $2^{\text {nd }}$ Preimage & (improving [5] w/ Sect. 4.3] & 10 & $2^{416}$ & $2^{64}$ & $2^{464}$ & $2^{64}$ \\
\hline $2^{\text {nd }}$ Preimage & (improving [5] w/ Sect.4.3) & 10 & $2^{384}$ & $2^{128}$ & $2^{448}$ & $2^{128}$ \\
\hline Collision $^{1}$ & (extension of this work) 5 & 14 & $2^{192}$ & $2^{128}$ & \multicolumn{2}{|c|}{$N / A$} \\
\hline Preimage $^{1}$ & (extension of this work) [5] & 14 & $2^{384}$ & $2^{128}$ & \multicolumn{2}{|c|}{$N / A$} \\
\hline Preimage $^{1}$ & (extension of this work) 5 & 14 & $2^{448}$ & - & \multicolumn{2}{|c|}{$N / A$} \\
\hline
\end{tabular}

${ }^{1}$ Chosen salt attacks 


\section{Acknowledgements}

We would like to thank the members of the Graz ECRYPT meeting. Especially, we would like to express our gratitude to Emilia Käsper, Christian Rechberger, Søren S. Thomsen, and Ralf-Philipp Weinmann for the inspiring discussions. We are grateful to the Lesamnta team, and especially to Hirotaka Yoshida, for helping us with this research. We would also like to thank the anonymous referees for their comments.

\section{References}

1. Biham, E., Dunkelman, O.: The SHAvite-3 Hash Function. Submission to NIST (2008)

2. Bouillaguet, C., Dunkelman, O., Fouque, P.A., Leurent, G.: Another Look at Complementation Properties. In: Hong, S., Iwata, T. (eds.) FSE 2010. LNCS, vol. 6147, pp. 347-364. Springer, Heidelberg (2010)

3. Bouillaguet, C., Dunkelman, O., Leurent, G., Fouque, P.A.: Attacks on Hash Functions based on Generalized Feistel - Application to Reduced-Round Lesamnta and SHAvite-3512. Cryptology ePrint Archive, Report 2009/634 (2009), http://eprint.iacr.org/

4. Mendel, F., et al.: A preimage attack on 8-round SHAvite-3-512. Graz ECRYPT meeting (May 2009)

5. Gauravaram, P., Leurent, G., Mendel, F., Naya-Plasencia, M., Peyrin, T., Rechberger, C., Schläffer, M.: Cryptanalysis of the 10-Round Hash and Full Compression Function of SHAvite-3-512. In: Bernstein, D.J., Lange, T. (eds.) AFRICACRYPT 2010. LNCS, vol. 6055, pp. 419-436. Springer, Heidelberg (2010)

6. Hirose, S., Kuwakado, H., Yoshida, H.: SHA-3 Proposal: Lesamnta. Submission to NIST (2008)

7. Khovratovich, D., Biryukov, A., Nikolic, I.: Speeding up Collision Search for ByteOriented Hash Functions. In: Fischlin, M. (ed.) CT-RSA 2009. LNCS, vol. 5473, pp. 164-181. Springer, Heidelberg (2009)

8. Lai, X., Massey, J.L.: Hash Functions Based on Block Ciphers. In: Rueppel, R.A. (ed.) EUROCRYPT 1992. LNCS, vol. 658, pp. 55-70. Springer, Heidelberg (1993)

9. Van Le, T., Sparr, R., Wernsdorf, R., Desmedt, Y.: Complementation-Like and Cyclic Properties of AES Round Functions. In: Dobbertin, H., Rijmen, V., Sowa, A. (eds.) AES 2005. LNCS, vol. 3373, pp. 128-141. Springer, Heidelberg (2005)

10. Hirose, S., Kuwakado, H., Yoshida, H.: Security Analysis of the Compression Function of Lesamnta and its Impact (2009) (available online) 